\title{
Retraso diagnóstico de la infección por el Virus de Inmunodeficiencia Humana
}

\section{Diagnostic delay of infection with Human Immunodeficiency Virus}

\author{
Martha Gómez Álvarez ${ }^{1}$ Mónica Hanna Lavalle ${ }^{2}$ Concepción Amador Ahumada ${ }^{3}$ (i)
}

Recibido para publicación: Febrero 15 de 2019 - Aprobado para publicación: Junio 5 de 2019

\begin{abstract}
RESUMEN
Objetivo. Analizar el comportamiento de la oportunidad diagnóstica evaluando Retrasos Diagnósticos (RD) y diagnósticos con Presentación en Etapa Avanzada (PEA) en casos VIH/ SIDA. Materiales y metodos. Estudio descriptivo retrospectivo de diagnósticos de la infección por el virus del VIH en instituciones de atención en salud. Se incluyeron los casos nuevos o incidentes diagnosticados entre el 1 enero 2011 a 31 de diciembre de 2016. Se rotuló como Retraso Diagnóstico (RD) aquellos que obtuvieron CD4 menor de 350, Presentación con Enfermedad Avanzada (PEA) por CD4 menor de 200, y una categoría adicional de CD4 menor de 500 utilizada como patrón de referencia. Resultados. Se ingresaron 188 incidentes todos con resultados de recuento de linfocitos CD4; la mediana de edad fue de 30 (Rango Intercuartil 23-40), mediana de CD4 de 230. En cuanto a la interpretación de los retrasos $43.6 \%$ de los casos presentó PEA, 68.6\% clasificó para RD. La proporción de RD y PEA fue mayor entre los hombres y en los grupos etarios de mayor edad; según el modo de transmisión predominó el sexual, siendo el RD y PEA más frecuente entre Hombres que tienen Sexo con Hombres $(\mathrm{HSH})$ contrastando con lo observado en otros estudios, situación que denota dificultades de focalización de los sujetos en riesgo. Conclusión. Los casos que están siendo diagnosticados en la fase final corresponden a individuos de mayor edad, de sexo masculino con antecedentes de prácticas de $\mathrm{HSH}$; los hallazgos permiten identificar factores que influyen en la falta de oportunidad para el diagnóstico, falencias la gestión en salud pública en lo relacionado con la prevención y control del VIH en poblaciones claves.
\end{abstract}

Palabras clave: VIH, Presentación con Enfermedad Avanzada, Retraso Diagnóstico, Presentación Tardía.

\begin{abstract}
Objective. To analyze the behavior of the diagnostic opportunity by evaluating Diagnostic Delays (RD) and diagnoses with Advanced Stage Presentation (PEA) in HIV / AIDS cases. Materials and methods. Retrospective descriptive study of diagnoses of infection by the HIV virus in health care institutions. New cases or incidents diagnosed between January 1, 2011 and December 31, 2016 were included. Diagnostic delay (RD) was labeled as those that obtained CD4 less than 350, Presentation with Advanced Disease (PEA) by CD4 less than 200, and an additional category of CD4 less than 500 used as reference standard. Results. 188 incidents were entered all with results of CD4 lymphocyte count; the median age was 30 (Interquartile range 23-40), median CD4 of 230. Regarding the interpretation of the delays $43.6 \%$ of the cases presented PEA, $68.6 \%$ classified for RD. The proportion of RD and PEA was higher among men and in the older age groups; according to the mode of transmission, sexuality predominated, with the RD and PEA being more frequent among Men who have Sex with Men (MSM), in contrast to what has been observed in other studies, a situation that denotes difficulties in targeting subjects at risk. Conclusion. The cases that are being diagnosed in the final phase correspond to older individuals, male with a history of MSM practices; the findings allow to identify factors that influence the lack of opportunity for diagnosis, failures in public health management in relation to the prevention and control of HIV in key populations.
\end{abstract}

Keywords: HIV, Presentation with Advanced Disease, Delayed Diagnosis, Late Presentation.

\section{Citación (Vancouver) \\ Gómez AM, Hanna LM, Amador AC. Retraso diagnósti- co de la infección por el virus de Inmunodeficiencia Humana. Rev Avances en Salud; 2019. (3)1:34-44. DOI: $\underline{10.21897 / 25394622.1764}$}

(C) 2019. Universidad de Cordoba. Este es un artículo de acceso abierto distribuido bajo los términos de la licencia Creative Commons Attribution License, que permite el uso ilimitado, distribución y reproducción en cualquier medio, siempre que el autor original y la fuente se acreditan.

\footnotetext{
1 M.Sc. (c) en Salud Pública. Auditora de calidad EPS-S Comfacor - Córdoba. Colombia.

${ }^{2}$ M.Sc. Administración de Organizaciones. Facultad Ciencias de la Salud. Universidad de Córdoba, Colombia

${ }^{3}$ M.Sc. en Enfermería. Facultad Ciencias de la Salud. Universidad de Córdoba, Colombia. Correspondencia: conceamador@yahoo.es
} 


\section{INTRODUCCIÓN}

La infección del Virus de Inmunodeficiencia Humana (VIH) durante los 37 años transcurridos de la pandemia sigue siendo uno de los problemas de Salud Pública más importantes en el mundo, a partir de los primeros casos que fueron identificados en 1981, la epidemia se ha cobrado más de 35 millones de vidas, a finales de 2016 se estima que un millón de personas fallecieron en el mundo por causas relacionadas con este virus (1).

Según el último reporte de la Organización Mundial de la Salud a diciembre de 2016, había alrededor de 37,6 millones de personas infectadas por $\mathrm{VIH}$ en el mundo, y en ese año se produjeron 1,8 millones de nuevas infecciones y $53 \%$ de las personas que vivían con el virus estaban recibiendo tratamiento (1).

La infección por el virus pasa por tres fases: una aguda que se manifiesta de dos a cuatro semanas, la crónica o de latencia que dura aproximadamente de 5 a 7 años y la fase final que es la más grave de la infección en la cual gran parte del sistema inmunitario ha sido destruido y en consecuencia el cuerpo ha mermado su capacidad de defensa contra las infecciones oportunistas y el cáncer. En los últimos 10 años la vigilancia de la infección por el virus de inmunodeficiencia adquirida ha aportado conocimiento de la epidemia y ha permitido adoptar eficientes y oportunas intervenciones de salud pública de utilidad para controlar la exposición a los factores de riesgo de la epidemia, el diagnóstico precoz y tratamiento oportuno (2).

En Europa occidental cerca del 50\% de los individuos recién diagnosticados con VIH son casos confirmados en una etapa tardía de la enfermedad e ingresan a la asistencia sanitaria tarde (es decir, con un recuento de CD4 $\leq$ $350 \mu \mathrm{L})$. En España como en el resto de países europeos se estima que cerca del $30 \%$ de las personas que conviven con el virus no están diagnosticadas, varios estudios españoles pu- blicados muestran que el porcentaje de pacientes con retraso diagnostico oscila entre el $29 \%$ y el $56 \%(3)$.

Según la definición de Retraso Diagnostico (RD) consensuada en el 2011 por el grupo de trabajo de Consenso Europeo se entiende como Presentadores Tardíos a las personas que son diagnosticadas o inician el procedimiento de atención con un recuento de linfocitos T CD4+ al diagnóstico $\leq 350$ células por micro litro o en ocasiones por la presencia de un evento definitorio de SIDA. También esta definición incluye la "Presentación con Enfermedad Avanzada" (PEA) que aplica cuando los linfocitos CD4+ en el momento diagnóstico son $\leq 200$ células por micro litro o la presencia de un evento definitorio de Sida (4).

En cuanto al VIH/SIDA, Colombia ocupó el cuarto lugar entre los países de Latinoamérica con mayor aumento de la incidencia de la infección lo que podría estar relacionado con la reemergencia de la infección. Según datos de la Cuenta de Alto Costo de Colombia un total de 83.551 registros de nuevos casos se encontraban reportados con corte a 31 de enero de 2017; de esta cifra, 9.396 son nuevos casos (5).

El retraso diagnóstico de la infección por VIH y la demora en el inicio del tratamiento antirretroviral son considerados condicionantes básicos que aumentan el riesgo de progresión de la enfermedad y de mortalidad temprana; adicionalmente desde la perspectiva epidemiológica tal situación implica un impacto negativo pues se incrementa el riesgo de transmisión del virus debido al aumento de la carga viral de los pacientes no tratados $(4,6)$.

En tal sentido el diagnóstico temprano no solo permite tratamientos oportunos y manejo preventivo de oportunistas que comprometen la vida de los pacientes, sino que al conocer el status seropositivo y someterse a tratamiento se está impactando la epidemia evitando nuevos 
casos. Realizar el diagnóstico temprano es uno de los principales retos de la respuesta a la epidemia del VIH-sida $(7,8)$.

El presente artículo describe las características, los factores sociales y demográficos que fueron asociados con el Diagnóstico Tardío y Etapa avanzada de la infección por el VIH en una población afiliada al régimen subsidiado de salud, buscando sensibilizar a la población académica, a los entes territoriales y municipales sobre la importancia del diagnóstico precoz y las implicaciones que tiene para los individuos, la comunidad y la sociedad el retraso diagnóstico de la infección por VIH; los resultados permiten analizar situaciones que favorezcan los programas para la promoción de la salud, la prevención de la enfermedad, la vigilancia de los riesgos y la orientación de las acciones quienes viven con VIH para mejorar y/o mantener la salud individual y colectiva (9).

\section{MATERIALES Y MÉTODOS}

Se realizó un estudio descriptivo, transversal y retrospectivo en donde se analizó el retraso diagnóstico de la infección por el VIH como problema de salud pública en Córdoba (Colombia).

La unidad de análisis fue cada uno de los nuevos diagnósticos que fueron reportados por las instituciones de atención de los casos de VIH/ SIDA en el periodo de cinco años continuos comprendidos entre el 1 de enero de 2011 a 31 de diciembre de 2016. Para evaluar el estado inmunológico se utilizó la información acerca del recuento de linfocitos TCD4 al momento del diagnóstico del VIH/SIDA con cifras de CD4 $\leq 350$ células/ $\mu$ l o diagnosticadas con SIDA (independientemente del recuento de células CD4) se definieron como últimos presentadores (RD); las personas con CD4 $\leq 200$ células o en estadío SIDA (independientemente del recuento de células CD4), los cuales se definieron como Presentación con Enfermedad
Avanzada de VIH (PEA).

La fuente de información fue secundaria pues se utilizaron los datos contenidos en las historias clínicas de los usuarios del programa de $\mathrm{VIH} / \mathrm{SIDA}$ de una institución de salud de naturaleza privada, que funciona en la capital del departamento de Córdoba (Colombia) y es la entidad de referencia para tratamiento de la morbilidad por esta causa.

La población de estudio fueron todas las historias clínicas de los nuevos diagnósticos de infección por $\mathrm{VIH} / \mathrm{SIDA}$ en el periodo comprendido entre el 1 de enero del 2011 al 31 de diciembre del 2016.

Se incluyeron todos los casos con RD, PEA y DT que se confirmaron durante la ventana de observación; debido a lo anterior, el tamaño de muestra fue de 188 usuarios incidentes en cinco años, quienes se encontraban registrados en las bases de datos, de quienes se tomaron datos retrospectivos de las historias clínicas previo aval institucional; para la clasificación de los casos, se utilizaron los criterios de definición ya constituidos para diagnóstico tardío (DT) y los subgrupos no excluyentes "Retraso Diagnóstico" y "Presentación con Enfermedad Avanzada" que incluye el nivel de linfocitos CD4/ $\mu \mathrm{l}$ al diagnóstico menor a 200/ $\mu \mathrm{l}$; siendo el único parámetro clasificatorio el resultado del nivel de linfocitos T CD4/ $\mu$ l en el momento del diagnóstico.

Durante la duración del estudio, todos los documentos del expediente clínico estuvieron localizados en un área segura y bajo la custodia de la entidad de salud. La base de datos que contenía la información de interés estuvo bajo la responsabilidad de la directora y de la investigadora principal, ningún archivo que se utilizó para la recogida de datos o para su análisis posterior llevó datos de identificación de pacientes. Durante el proceso de captura de la información se garantizó la confidencialidad 
de los datos de todos los participantes del estudio y el cumplimiento de la normativa acerca de la ética de la investigación contenida en la Resolución 8430 de 1993 de Colombia.

La información se consolidó en una base de datos estructurada en Microsoft Excel versión 1.0 que fue depurada; el reporte y análisis estadístico se realizó con el programa SPSS Statistics v.22, para Windows. Para describir las variables categóricas se utilizó estadística descriptiva; así mismo se aplicaron medidas de tendencia central y medidas de dispersión aplicadas al comportamiento de los Linfocitos T CD4 iniciales con su rango, promedio, mediana y cuartiles al igual que su medida de dispersión.

\section{RESULTADOS}

Entre los 188 casos incidentes con retraso diagnóstico, se observó una mediana en edad de 30 años el rango de edad estuvo entre 1 y 61 años; el espacio intercuartil estuvo entre los 23 a 40 años, que se interpreta en el sentido que $25 \%$ de los casos con RD son menores de 23 años y $25 \%$ eran mayores de 40 años. (Tabla 1).

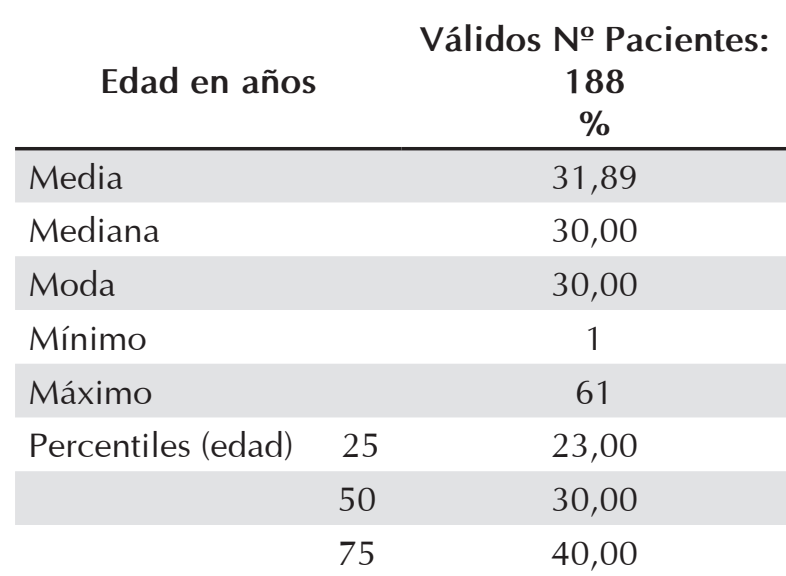

Tabla 1. Mediana de edad en años. Fuente: Base de datos IPS.

Los grupos etarios con más personas infectadas por el virus, con RD, PEA y DT se ubicaron a partir de los 20 años de edad. (Gráfica 1).

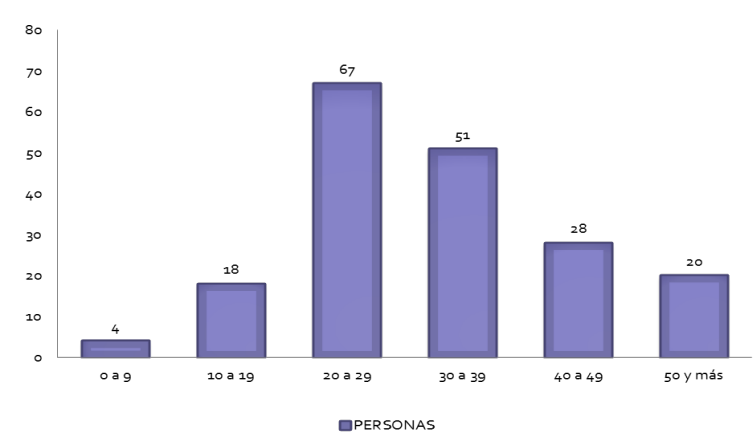

Gráfica 1. Distribución de los casos por grupos etareos. Fuente: Bases de datos IPS.

Características relacionadas con el retraso diagnóstico. Las características del grupo diagnosticado con retraso fue de $56.9 \%$ hombres y $43.1 \%$ mujeres, en hombres la mediana fue de 33 años (rango intercuartil de 25-42) y en mujeres la mediana fue de 26 (rango intercuartil de 22-34). En cuanto a la vía de transmisión, $97.4 \%$ fueron infectados por vía sexual, especialmente por relación heterosexual $(78.7 \%)$, seguido de $\mathrm{HSH}$ con $18.6 \%$ (Tabla 2 ).

\section{Sexo}

\begin{tabular}{|c|c|c|}
\hline Femenino & 81 & $43,1 \%$ \\
\hline Masculino & 107 & $56,9 \%$ \\
\hline \multicolumn{3}{|c|}{ Mediana de la Edad en años } \\
\hline Total & 30 & $23-40$ \\
\hline Femenino & 26 & $22-34$ \\
\hline Masculino & 33 & $25-42$ \\
\hline \multicolumn{3}{|c|}{ Modo de transmisión } \\
\hline $\begin{array}{l}\text { Transmisión } \\
\text { Sexual }\end{array}$ & 183 & $97,4 \%$ \\
\hline $\begin{array}{l}\text { Transmisión } \\
\text { Vertical }\end{array}$ & 4 & $2,1 \%$ \\
\hline
\end{tabular}

Tabla 2. Características relacionadas con el retraso diagnóstico. Fuente: Base de datos IPS.

La transmisión sexual continúa siendo el mecanismo conocido más importante para contagio con el virus en los nuevos casos con $97,4 \%$, el retraso diagnóstico se presentó en $68 \%$ en 
el modo de transmisión heterosexual, el modo de trasmisión vertical presentó 25\% para retraso diagnóstico, y solo un paciente desconocía como fue su modo de infección con el virus.

Cabe destacar que 8 de cada 10 casos incidentes se infectaron con el modo de contacto heterosexual lo cual expresa el nivel de riesgo de contagio con el virus entre personas residentes en el departamento; en el contraste con la edad, se observa que este fenómeno corresponde a los adolescentes y adultos jóvenes los cuales tienen muy poca percepción del riesgo

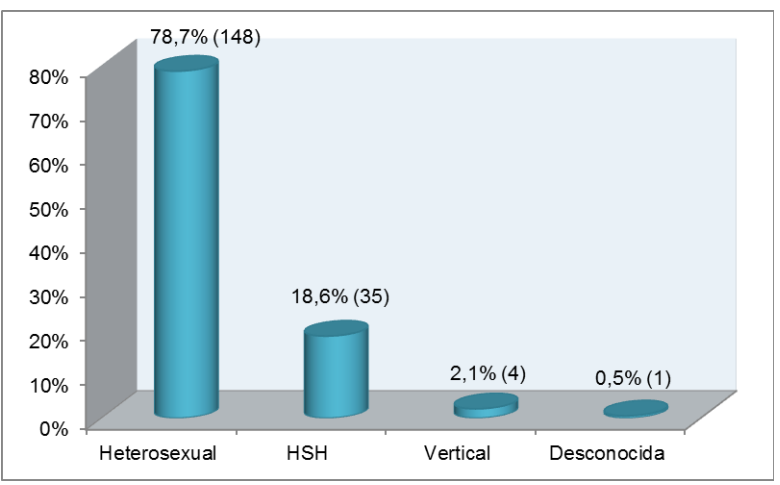

Gráfica 2. Modo de transmisión de los casos de RD, PEA y DT. Fuente: Base de datos IPS. y acuden al sexo casual, esporádico o libre en busca de diversión (Gráfica 2).

Comportamiento de la oportunidad diagnostica evaluando Retrasos Diagnósticos (RD) y diagnósticos con Presentación en Etapa Avanzada (PEA). Todos los pacientes diagnosticados en este período (100\%) tenían CD4 de ingreso, con una mediana de 230 células/ $\mu$, el CD4 más bajo fue de 7 y el mayor de 2245/ $\mu \mathrm{l}$, el 25\% con menos de $71 / \mu \mathrm{l}$ CD4, y 25\% con CD4 mayor de

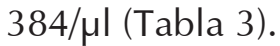

Mediana de LCD4

\begin{tabular}{lcc}
\hline N Validos & & 188 \\
N Perdidos & & 0 \\
Media & & 276,91 \\
Mediana & & 230,50 \\
Mínimo & 25 & 7 \\
Máximo & 50 & 2245 \\
\hline Percentiles & 75 & 70,25 \\
& & 230,50 \\
& & 384,50
\end{tabular}

Tabla 3. Mediana de Linfocitos T CD4 inicial. Fuente: Base de datos IPS.

Al graficar la mediana de linfocitos CD4 en los últimos años se observa un incremento entre los años 2011 y 2012; alcanzando su punto más bajo en el año 2014; las cifras de 2015 evidencian una tendencia al ascenso aunque se conservó inferior a la presentada al inicio del periodo observado (Gráfica 3). 


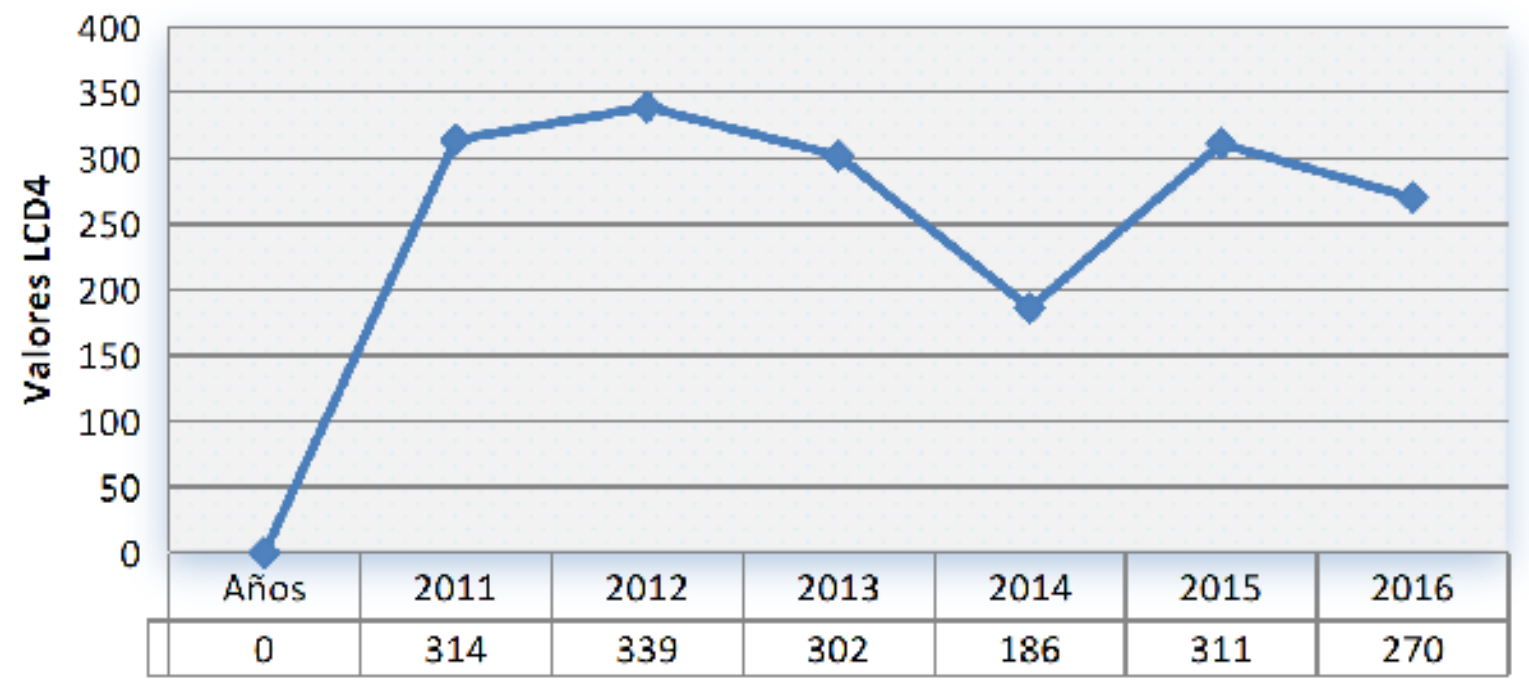

Gráfica 3. Mediana de Linfocitos T CD4 al momento del diagnóstico por año. Fuente: Base de datos IPS.

De los 188 casos diagnosticados en este período 82(43.6\%) estaban con Presentación con Enfermedad Avanzada (PEA), 129 individuos (68.6\%) tuvieron Retraso Diagnóstico (RD); y del total de casos incidentes 166 sujetos (88.2\%) presentaban menos de 500/ $\mu$ l CD4, esto es que fueron diagnosticados de forma oportuna.

\begin{tabular}{|c|c|c|c|c|c|}
\hline \multirow{2}{*}{ Características } & \multirow{2}{*}{$\begin{array}{c}N \text { casos con } \\
\text { Linfocitos T } \\
\text { CD4 (N y } \\
\%)\end{array}$} & \multicolumn{2}{|c|}{ Enfermedad Avanzada } & \multicolumn{2}{|c|}{ Diagnóstico Tardío } \\
\hline & & $N$ & $\%$ & $N$ & $\%$ \\
\hline $\begin{array}{l}\text { Sexo: } \\
\text { Femenino } \\
\text { Masculino }\end{array}$ & $\begin{array}{l}81(100.0) \\
107(100.0)\end{array}$ & $\begin{array}{l}29 \\
53\end{array}$ & $\begin{array}{l}35.8 \\
49.5\end{array}$ & $\begin{array}{l}51 \\
78\end{array}$ & $\begin{array}{l}63.0 \\
72.9\end{array}$ \\
\hline $\begin{array}{l}\text { Edad en años: } \\
\begin{array}{l}0 \text { a } 9 \\
10 \text { a } 19 \\
20 \text { a } 29 \\
30 \text { a } 39 \\
40 \text { a } 49 \\
50 \text { y más }\end{array}\end{array}$ & $\begin{array}{l}4 \\
18 \\
67 \\
51 \\
28 \\
20\end{array}$ & $\begin{array}{l}0 \\
3 \\
25 \\
24 \\
17 \\
13\end{array}$ & $\begin{array}{l}0.0 \\
16,7 \\
37,3 \\
47,1 \\
60,7 \\
65,0\end{array}$ & $\begin{array}{l}1 \\
9 \\
40 \\
35 \\
24 \\
20\end{array}$ & $\begin{array}{l}25,0 \\
50,0 \\
59,7 \\
68,6 \\
85,7 \\
100,0\end{array}$ \\
\hline
\end{tabular}




\begin{tabular}{|ll|ll|ll|} 
Año de Diagnóstico & & & & \\
2011 & 17 & 4 & 23,5 & 12 & 70,6 \\
2012 & 20 & 4 & 20,0 & 9 & 45,0 \\
2013 & 37 & 12 & 32,4 & 26 & 70,3 \\
2014 & 38 & 27 & 71,1 & 32 & 84,2 \\
2015 & 29 & 15 & 51,7 & 20 & 69,0 \\
2016 & 47 & 20 & 42,6 & 30 & 63,8 \\
& & & & & \\
Modo de Transmi- & & & & & \\
sión & 143 & 61 & 41.2 & 101 & 68.2 \\
Heterosexual & 40 & 0 & 57.1 & 26 & 74.3 \\
HSH & 4 & 1 & 0.0 & 1 & 25.0 \\
Vertical & 1 & & 100,0 & 1 & 100.0 \\
Desconocida & 188 & & & 68.6 \\
\hline Total & & & & \\
\hline
\end{tabular}

Tabla 3. Estadíos de la enfermedad. Fuente: Bae de datos IPS.

Al revisar los hallazgos por categorías se identifica que en hombres se presentó mayor porcentaje de PEA que en mujeres $(49.5 \%$ y $35.8 \%$ respectivamente), al igual que Retraso Diagnóstico $(72.9 \%$ hombres y $63.0 \%$ mujeres); en el grupo de usuarios diagnosticados con oportunidad con conteo de linfocitos CD4 menor de $500 / \mu \mathrm{l}$, en este grupo la distribución de la proporción es más homogénea $(89.7 \%$ en hombres y $86.4 \%$ en mujeres.

Al revisar edad se observa una relación directamente proporcional que evidencia que a medida que la edad aumenta, también se incrementa el porcentaje de pacientes tanto en RD como en PEA, observándose que $65 \%$ de las personas con 50 o más años clasificaron para Presentación con Enfermedad Avanzada y $100 \%$ de personas de este mismo rango de edad presentan Retraso Diagnóstico (RD)

No se observa un patrón claro de Diagnósticos Tardíos o Presentación con Enfermedad Avan- zada al revisar por años de diagnóstico pero si se observa un leve descenso en ambos grupos a partir del año 2013. El modo de transmisión heterosexual es el predominante representando $68.2 \%$ de los RD y $41.2 \%$ de los PEA; en esta categoría el grupo de $\mathrm{HSH}$ presentó $74.3 \%$ de RD y $57.1 \%$ de PEA, y en transmisión vertical $25 \%$ tuvo RD y ninguno se diagnosticó con PEA. En solo un caso no se pudo establecer con precisión la forma de transmisión el cual se diagnosticó con PEA y por supuesto con RD.

\section{DISCUSIÓN}

En este estudio que la proporción de RD (menor de $350 / \mu \mathrm{l}$ ) de $68.6 \%$ y VIH con PEA (menor de 200/ $\mathrm{Ml}$ CD4) fue de $43.6 \%$, y menores de 500 de $88.2 \%$. Estas proporciones son mayores a las encontradas en el estudio de Celesia (2016) quien encontró respectivamente (RD 55,6\%, PEA 35\%); así mismo son comparables con los hallazgos de Rivero (2014 RD 55\%). (10, 11) 
Los resultados del estudio RD (menor de 350) de $68.6 \%$ y $\mathrm{VIH}$ con PEA (menor de $200 / \mu \mathrm{l}$ CD4) fue de $43.6 \%$ hallazgos que difieren con los encontrados por Maquera (2016) con un DT $(82,6 \%)$, como de EA (64,5\%), (PEA 29,4\%RD $48,1)$ difiere también con los resultados de Agaba (2014) (RD 85,6\%y PEA 63\%). Entre los hallazgos se destaca que $11.8 \%$ fueron diagnosticados con CD4 mayor de 500/ $\mu$ l lo cual es un porcentaje muy bajo de diagnóstico precoz para intervenciones oportunas $(12,13,14)$.

Los resultados son interesantes por cuanto en Colombia se implementó la GPC basada en la evidencia publicada en 2015 que establece que en la fase diagnóstica privilegia la oportunidad en el diagnóstico con algoritmos ágiles y enfocados en la captación de pacientes con dos pruebas presuntivas, en el corto tiempo con lo que se espera observar un acortamiento en los tiempos de inicio de tratamiento a los pacientes, sin embargo en los resultados del presente estudio se aprecia que la GPC referida no se estaría cumpliendo a cabalidad en todos los casos, lo cual acarrea dificultades en el control de la epidemia de esta enfermedad (15).

Para resolver este problema que generará un impacto negativo alto sobre el control de la epidemia de VIH/SIDA, debería considerarse, la necesidad de implementar o incrementar el tamizaje universal por lo menos en los servicios de urgencia, previo trabajo de sensibilización a todo el personal de salud de confidencialidad, estigma y responsabilidad legal de los funcionarios de salud para reducir el RD y la PEA en esta región de Colombia (15).

En el presente estudio las características demográficas de mayores frecuencias fueron las edades mayor o igual de 30 años, el sexo masculino y el mecanismo de transmisión sexual en $\mathrm{HSH}$, mismas características que se relacionaron con una mayor frecuencia de RD y de PEA con hallazgos similares a los obtenidos 'por Baral et al (2007). Por sexo se observa una ma- yor frecuencia de RD y de PEA en hombres que en mujeres, lo cual puede ser producto que en casi la tercera parte de diagnósticos de mujeres se hizo durante el embarazo pues el protocolo demanda de la realización rutinaria de la prueba de VIH. Este predominio en el sexo masculino ha sido hallado en otros estudios como los de Kilmarx (2009), García (2012) en los que se destaca que la enfermedad predomina en el sexo masculino (16-19).

Al revisar las relaciones entre el PEA y RD (57.1\% y $74.3 \%$ respectivamente) y el modo de transmisión se observa que la distribución privilegia como vía de transmisión la sexual en particular a los hombres que tienen sexo con hombres contrastando con estudios donde se encontró menor frecuencia de estas dos categorías en transmisión heterosexual, y la transmisión HSH fue más baja debido al efecto de los mecanismos y estrategias educativas de focalización y control del riesgo. $(16,17)$

Los hallazgos acerca de la transmisión heterosexual, y la transmisión HSH fue más baja comparada con los hallazgos de Maquera (HSH 8\% y heterosexual $12 \%$ ) y Camoni $(\mathrm{HSH} 44,3 \%$ heterosexual $61,1 \%$ ) debido a que en estos estudios se identificó la mayor frecuencia de RD y PEA en población heterosexual, la cual sucede de tal modo por la menor percepción del riesgo entre heterosexuales, y por una mayor percepción de ese riesgo en $\mathrm{HSH}$, en tanto que por algún motivo en el contexto del presente estudio los $\mathrm{HSH}$ esto no ocurre, de lo cual se deduce que existe una pobre difusión en el reconocimiento y conciencia del riesgo en ellos $(2,12)$.

El comportamiento de la distribución de RD y PEA en relación con la edad; evidencia una relación categórica positiva que indica que a medida que se aumenta la edad es mayor el nivel de RD y la PEA; este dato se relaciona probablemente con una menor percepción del riesgo de contagio que tienen las personas de mayor 
edad, así como la poca suspicacia o sospecha clínica por parte del personal sanitario que al parecer no asocia la posibilidad de encontrar esta patología en personas con edades mayores; por tanto esta carencia de sospecha clínica impide la realización de las pruebas requeridas para descartarla, lo cual indica que en la zona, se está desconociendo la tendencia del comportamiento mundial de expansión de la epidemia VIH/SIDA que muestra unas mayores prevalencias en las personas de mayor edad, debido a la focalización de las actividades y programas dirigidos a los jóvenes.

Ante esta evidencia que se presenta en los resultados, se requiere o demanda de la necesidad de implementar estrategias que permitan aumentar y mejorar el acceso a las pruebas de tamizaje y diagnóstica a las poblaciones con mayor vulnerabilidad y por tanto mayor riesgo como son los $\mathrm{HSH}$, personas que ejercen la prostitución, personas inmigrantes, poblaciones móviles y minorías étnicas, para los adultos mayores se requieren medios alternativos de oferta de la prueba $(2,8,9)$.

Es preocupante la mayor representación de RD y PEA en población HSH ya que va en contraposición a lo observado en otros países, denotando una clara deficiencia en el abordaje de poblaciones de alto riesgo de infección por VIH pues la focalización parece que está orientada hacia los varones homosexuales y hacia las mujeres en gestación; descuidando otros grupos igualmente vulnerables, lo cual representa en este caso un factor importante en el retraso diagnóstico; es prudente aclarar que en otros países los $\mathrm{HSH}$ se realizan de forma voluntaria la prueba Elisa al menos una vez al año, como indicación precisa de la conciencia del riesgo $(8,9,15)$.

\section{CONCLUSIONES}

La accesibilidad a las pruebas continua siendo un gran problema y es que a los pacientes no se les puede ordenar la prueba sin previo consentimiento informado (pre-test), lo cual requiere personal entrenado y certificado, con frecuencia no se ordena porque no hay quien haga la asesoría pre-test, esto se ha obviado un poco en las embarazadas con la obligatoriedad de realizar las pruebas, sin embargo la cobertura de pruebas a embarazadas no es universal y aun en Colombia como gran parte de Latinoamérica no supera el $80 \%$.

Se ha avanzado poco en el diagnóstico y tratamiento temprano de esta enfermedad por lo cual se deben aumentar las acciones de prevención primaria, con intervenciones focalizada a las poblaciones en mayor riesgo, a fin de lograr una reducción soportada y enérgica en el número de nuevas infecciones de VIH anuales.

A pesar de todos los esfuerzos y campañas seguimos observando una gran proporción de Diagnósticos Tardío y Presentación con Enfermedad Avanzada, comprometiendo la salud de los pacientes y aumentando el riesgo de mortalidad y complicaciones incapacitantes.

Se requiere ampliar estudios de oportunidad en el diagnóstico para sensibilizar y presionar a los niveles de decisión en la necesidad de aumentar mecanismos de sensibilización del riesgo a la población vulnerable y a la general y al personal de salud para pensar en la patología y ordenar oportunamente las pruebas, es bien conocido el evento de pacientes que consultan gran cantidad de veces con síntomas premonitorios que son ignorados por el personal de salud. Se requiere reforzar la necesidad de serología de VIH ante la presencia de ciertas patologías y ante conductas de riesgo.

Se debe aumentar la cobertura de las pruebas diagnósticas de la infección por VIH y otras ITS para conseguir disminuir la fracción no diagnosticada, dar la formación adecuada entre los profesionales que prescriben la prueba a fin de que lo realicen de forma precoz, mediante la 
sospecha en la primo infección o en pacientes con síntomas indicativos de infección por $\mathrm{VIH}$, tanto en Atención Primaria como en Atención Especializada, así como en centros específicos, en áreas con una mayor prevalencia de enfermedad.

\section{RECOMENDACIONES}

Ampliar estudios de oportunidad en el diagnóstico para sensibilizar y presionar a los niveles de decisión en la necesidad de aumentar mecanismo de sensibilización del riesgo a la población; reforzando la necesidad de serología de VIH ante la presencia de ciertas patologías y ante conductas de riesgo.

Es importante que en Córdoba (Colombia) se realicen estudios cualitativos sobre el tema para reconocer la percepción del riesgo pues tal vez los usuarios ocultan estos riesgos por temor a la discriminación pues según las normativas nacionales es fácil acceder a los servicios diagnósticos.

\section{CONFLICTO DE INTERÉS}

Las autoras declaramos que no existe ningún tipo de conflicto de intereses ni por el equipo investigador, ni de ninguno de los participantes en el estudio.

Fuentes de financiación. El desarrollo del presente estudio no ha requerido fuentes de financiación externa

Implicaciones éticas. La responsabilidad de la base de datos se mantuvo en la entidad que suministró los datos; la base de datos que generó el proyecto de investigación se mantuvo anónima, sin ningún dato que permitiera la identificación alguna de pacientes por parte de terceros.

\section{REFERENCIAS}

1. Organización Mundial de la Salud. Estrategia mundial del sector de la salud contra el VIH 2016-2021: Hacia el fin del SIDA [Internet]. [Consultado 21 Dic 2016]. Disponible en: http://apps.who.int/iris/bitstream/10665/250574/1/WHO-HIV2016.05

2. Camoni $\mathrm{L}$, et al. "Late presenters among persons with a new HIV diagnosis in italy, 2010-2011". (2013). BMC public health 13.1281.

3. Rivero, Marcotegui M., et al. (2014). "Diagnóstico tardío de la infección por el virus de la inmunodeficiencia humana: oportunidades diagnósticas perdidas." Anales del Sistema Sanitario de Navarra. vol. 37. no. 3. gobierno de navarra. departamento de salud, 2014

4. Antinori A., et al. "Late presentation of HIV infection: a consensus definition." HIV medicine 12.1 (2011): 61-64.

5. Cuenta de Alto Costo. Boletín de información técnica especializada. Volumen $3 \mathrm{~N}^{\circ}$ 20, Dic 1 de 2017.

6. Sarria Urigüen L. Diagnóstico tardío de la infección por VIH: un problema en aumento Facultad de Medicina y Odontología. Universidad del País Vasco-Euskal Herriko Unibertsitatea.

7. Departamento de Salud y Servicios Humanos de Estados Unidos. Visión general de la infección por el VIH-sida. En el portal https://infosida.nih.gov/understanding-Hiv-aids/fact-sheets/19/46/las-fasesde-la-infección-por-el-VIH.

8. Plan de Acción para la Prevención y el Control de la infección por el VIH y las infecciones de Transmisión Sexual 20162021-55.o Consejo Directivo 68.a sesión 
del comité regional de la OMS. Para las américas Washington, D.C., EUA, del 26 al 30 de septiembre del 2016.

9. ONU-SIDA. Prevención de la infección por el VIH "Bajo la Lupa". Un análisis desde la perspectiva del sector de la salud en América Latina y el Caribe. Washington D.C.; 2017.

10. Celesia BM., et al. Late presentation of HIV infection: predictors of delayed diagnosis and survival in Eastern Sicily. Eur Rev Med Pharmacol Sci 17.16 (2013): 2218-2224.

11. Rivero Marcotegui M., et al. Diagnóstico tardío de la infección por el virus de la inmunodeficiencia humana: oportunidades diagnósticas perdidas. Anales del Sistema Sanitario de Navarra. Vol. 37. No. 3. Gobierno de Navarra. Departamento de Salud, 2014

12. Maquera AJ., et al. Diagnóstico tardío y enfermedad avanzada de VIH en pacientes adultos en un hospital de la seguridad social de Perú. Revista chilena de infectologia 33 (2016): 20-26.

13. Agaba P.A., et al. Patients who present late to HIV care and associated risk factors in Nigeria. HIV Medicine 15.7 (2014): 3964052
14. Noda Albelo AL, et al. Interpretación clínica del conteo de linfocitos T CD4 positivos en la infección por VIH. Revista Cubana de Medicina 52.2 (2013): 118-127.

15. Ministerio de Salud y Protección Social. Fondo de Población de Naciones Unidas. Guía de práctica clínica (GPC) basada en la evidencia científica para la atención de la infección por $\mathrm{VIH} /$ Sida en adolescentes (con 13 años de edad o más) y adultos. 2015. En portal web ministerial.

16. Baral S, Sifakis F, Cleghorn F, Beyrer C. Elevated risk for HIV infection among men who have sex with men in low- and middle-income countries 2000- 2006: a systematic review. PLoS Med. 2007 Dec; 4(12):e339.

17. Johnson WD, Diaz RM, Flanders WD, Goodman M, Hill AN, Holtgrave D, et al. Behavioral interventions to reduce risk for sexual transmission of HIV among men who have sex with men.

18. Kilmarx PH. Global epidemiology of HIV. Curr Opin HIV AIDS. 2009;(4):240-6.

19. García de Olalla P, Reyes JM, Caylà JA. Retraso diagnóstico de la infección por VIH. Rev. esp. sanid. penit. 2012 Jun; 14(1):2835 . 